EPJ Web of Conferences 81, 01002 (2014)

DOI: $10.1051 /$ epjconf/ 20148101002

(C) Owned by the authors, published by EDP Sciences, 2014

\title{
Photoproduction of Mesons on Quasi-Free Nucleons
}

\author{
I. Keshelashvili ${ }^{1,2, a}$ \\ ${ }^{1}$ Department of Physics, University of Basel, Klingelbergstrasse 82, CH-4056 Basel, Switzerland \\ ${ }^{2}$ Institut für Kernphysik, Forschungszentrum Jülich, 52425 Jülich, Germany
}

\begin{abstract}
The investigation of excited baryon states is important to understand the underling nature/symmetries of hadronic matter. Historically, the first nucleon excitation experiments have been done using charged pion and kaon secondary beams. Later the antiproton-proton scattering has also been involved. However, since the beginning of the 90's meson photoproduction reactions have been considered as a powerful tool in baryon spectroscopy. In this contribution, we overview our experimental programs conducted at the bremsstrahlung photon beams of the MAMI accelerator in Mainz and the ELSA accelerator in Bonn. The results are differential and total cross sections for photoproduction of light neutral mesons and of meson pairs off quasi-free nucleons bound in the deuteron (and sometimes other light nuclei). The scientific programs of this experiments also include single and double polarization measurements as well.
\end{abstract}

\section{Introduction}

The modern experiments worldwide are using virtual or real photon beams and measure neutral and/or charged reaction products. The large solid-angle electromagnetic calorimeters, Crystal Ball/TAPS at MAMI (Mainz) and Crystal Barrel/TAPS at ELSA (Bonn) use real tagged-photon beams, the magnetic CLAS spectrometer used also mostly tagged photons (partly also electron scattering), and the A1 collaboration at MAMI concentrates completely on electron scattering. Unfortunately, the nonexistence of free neutrons requires the use of quasi-free neutrons as targets (bound in the deuteron or the other light nuclei). Despite the great efforts the physical parameters of many well established states remain uncertain. Moreover, the existence of strong isospin dependence of the electromagnetic transition amplitudes can only be studied with meson photoproduction off the neutron. The final goal of this activities is to collect (almost) complete data sets, allowing Partial-Wave Analysis (PWA) and the extraction of nucleon resonance properties without model dependent ambiguities.

\section{Experiment}

In the presented series of experimental results the measurements were performed at two different real photon beam facilities: The continuous wave accelerator complex MAMI $[1,2]$ at the University of Mainz, Germany. And second the Electron Stretcher Accelerator ELSA [3] at the University of Bonn, Germany. High energy photon beams were produced in both cases from a fixed energy

ae-mail: i.keshelashvili@unibas.ch 
(MAMI: $1.6 \mathrm{GeV}$, ELSA: $2.4 \mathrm{GeV}$ ) electron beam scattered on high $Z$ material like copper producing bremsstrahlung photons. A schematic view of the A2 experiment at MAMI is shown in figure 1.

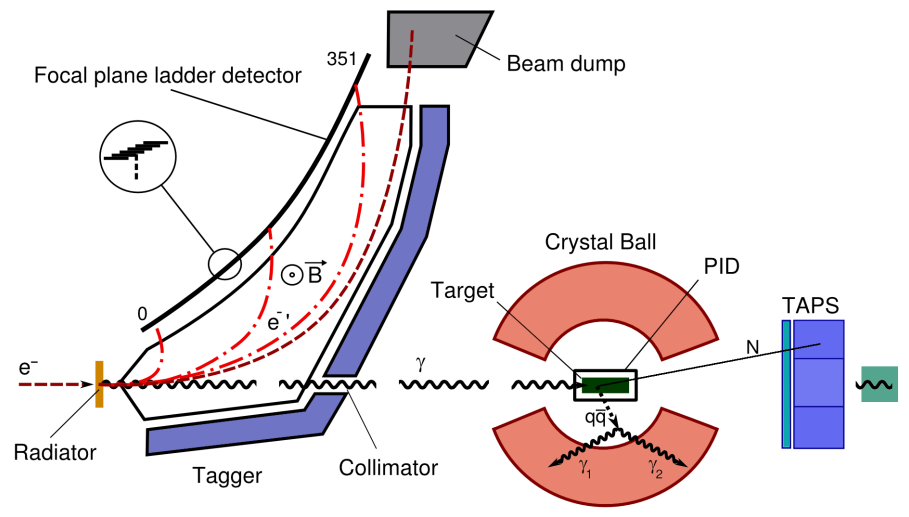

Figure 1. Schematic sketch of the A2 experimental setup at MAMI.

Since the majority of the presented results were measured with the A2 experiment, we will overview this particular setup in more detail and always indicate the difference between the two detectors. The energy of the photons in both experiments is determined by a momentum analysis of the scattered electron in a magnetic spectrometer (Glasgow Photon Tagger [4-6]). After collimation the beam impinged on the target (liquid deuterium or ${ }^{3} \mathrm{He}$ and transversely and longitudinally deuterated polarized buthanolin Mainz and liquid deuterium and longitudinally polarized frozen spin target in Bonn). The target was surrounded by a cylindrical plastic scintillator strip detector [7], which was used for charged particle identification and the spherical electromagnetic calorimeter Crystal Ball [8]. Similar to the A2 setup, for the charge particle identification inside the Crystal Barrel (surrounding the target) three layers of the intersecting scintillator fibre detector were used [12]. The Crystal Ball detector consists of $672 \mathrm{NaI}(\mathrm{Tl})$ crystals and covers $94 \%$ of $4 \pi$ steradians. The hole in the forward direction is closed by the TAPS detector $[9,10]$ which is made of $384 \mathrm{BaF}_{2}$ crystals $\left(378 \mathrm{BaF}_{2}+24\right.$ $\mathrm{PbWO}_{4}$ crystals in the ${ }^{3} \mathrm{He}$ experiment). In front of every crystal a thin plastic scintillator element is installed as a charged particle veto detector. At the ELSA electron synchrotron, the Crystal Barrel/TAPS electromagnetic calorimeter is installed. The Crystal Barrel consists of $1320 \mathrm{CsI}(\mathrm{Tl})$ [11] and 216 forward $\mathrm{BaF}_{2}[9,10]$ crystals with a polar angle coverage down to $1^{o}$ in a forward direction. As trigger condition, a deposited energy sum of $300 \mathrm{MeV}$ in the Crystal Ball and a total multiplicity of two or more hits was requested. In the case of the Crystal Barrel/TAPS detector, the only optimal trigger condition was the $\eta$ into $6 \gamma$ decay channel selection because the Crystal Barrel cannot trigger on neutral particles so that only photons in forward direction (Forward plug and TAPS) contributed to the trigger.

\section{Analysis}

The Mainz experiments used the $2 \gamma$ decays of the $\pi^{0} / \eta$ and the $6 \gamma$-decay of the $\eta$ (the Bonn experiments only the $6 \gamma$ decay of the $\eta$ ). Therefore, 2(6) neutral clusters +1 charged cluster or 3(7) neutral clusters were requested, respectively, for the Crystal Ball/TAPS experiment, see table 1. The mesons where identified in the invariant mass spectra (see figure 2) by applying a cut around the expected meson mass. Background coming from other channels was suppressed using cuts on the meson-nucleon 
MESON 2014 $-13^{\text {th }}$ International Workshop on Production, Properties and Interaction of Mesons

Table 1. Event selection criteria at A2 setup for the $\eta$ meson (in $\pi^{0}$ case only upper $(\gamma \gamma)$ line can be used) for exclusive and inclusive reaction identification. At CBELSA/TAPS only the lower $\left(3 \pi^{0}\right)$ condition can be used due to trigger system restrictions.

\begin{tabular}{c|c|c|c}
$\begin{array}{c}\text { decay } \\
\text { channel }\end{array}$ & $\sigma_{p}$ & $\sigma_{n}$ & $\sigma_{\text {incl }}$ \\
\hline$\eta\left(\pi^{0}\right) \rightarrow 2 \gamma$ & $2 n_{\eta / \pi^{0}} \& 1 c_{p}$ & $2 n_{\eta / \pi^{0}} \& 1 n_{n}$ & $2 n\left|\sigma_{p}\right| \sigma_{n}$ \\
$\eta \rightarrow 3 \pi^{0} \rightarrow 6 \gamma$ & $6 n_{\eta} \& 1 c_{p}$ & $6 n_{\eta} \& 1 n_{n}$ & $6 n\left|\sigma_{p}\right| \sigma_{n}$
\end{tabular}

coplanarity condition and the missing mass. Examples for the event selection for $\eta$ production for both exclusive and inclusive channels are shown in the table 1.

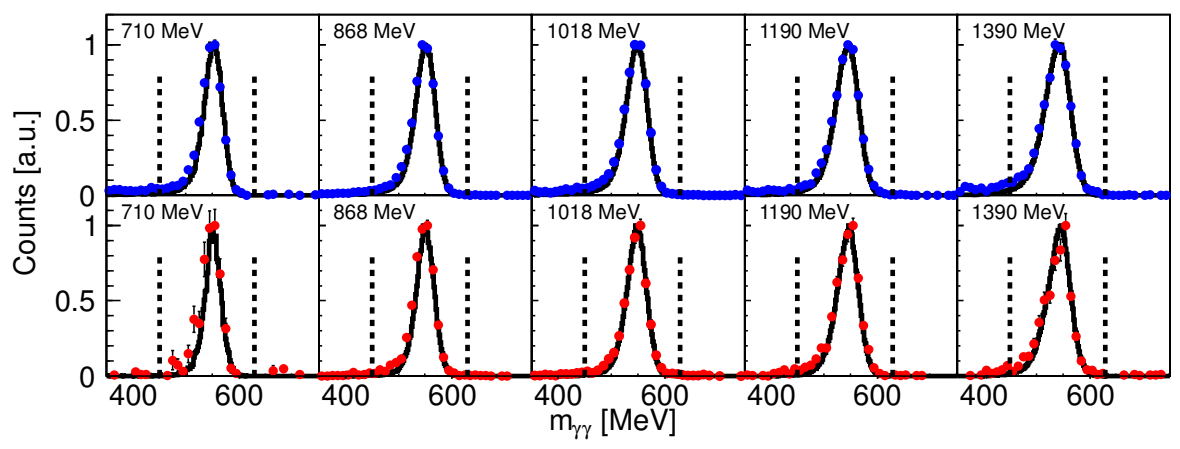

Figure 2. The invariant mass distribution in the case of $\eta$ production off ${ }^{3} \mathrm{He}$ target. Blue points data from quasi-free proton and red points quasi-free neutron data. The solid line Monte-Carlo simulation normalised on data points. The dotted vertical lines indicate $\eta$-meson mass cut.

\section{$4 \pi^{0}$-photoproduction off quasi-free nucleons}

The single $\pi^{0}$ photoproduction off quasi-free neutrons has never been investigated with dedicated measurements. The reason was the isospin decomposition of the cross section was based on the "precise" measurement of the charged and neutral pion production [13] off hydrogen targets and charged pion production off quasi-free neutrons. For pion photoproduction (isovector mesons) three independent amplitudes contribute to the reaction mechanism: the isoscalar part, the isospin dependent and the isospin changing amplitude [14].

$$
\begin{array}{ll}
A\left(\gamma p \rightarrow \pi^{+} n\right)=-\sqrt{\frac{1}{3}} A^{V 3}+\sqrt{\frac{2}{3}}\left(A^{I V}-A^{I S}\right) & A\left(\gamma n \rightarrow \pi^{-} p\right)=+\sqrt{\frac{1}{3}} A^{V 3}-\sqrt{\frac{2}{3}}\left(A^{I V}+A^{I S}\right) \\
A\left(\gamma p \rightarrow \pi^{0} p\right)=+\sqrt{\frac{2}{3}} A^{V 3}+\sqrt{\frac{1}{3}}\left(A^{I V}-A^{I S}\right) & A\left(\gamma n \rightarrow \pi^{0} n\right)=+\sqrt{\frac{2}{3}} A^{V 3}+\sqrt{\frac{1}{3}}\left(A^{I V}+A^{I S}\right)
\end{array}
$$

After the measurement of three reactions of the four given in equation 1, it was considered that the reaction was parametrised with enough precision. Because of strong nonresonant background terms for the charged pion production, one can expect a cleaner amplitude extraction for neutral pion 


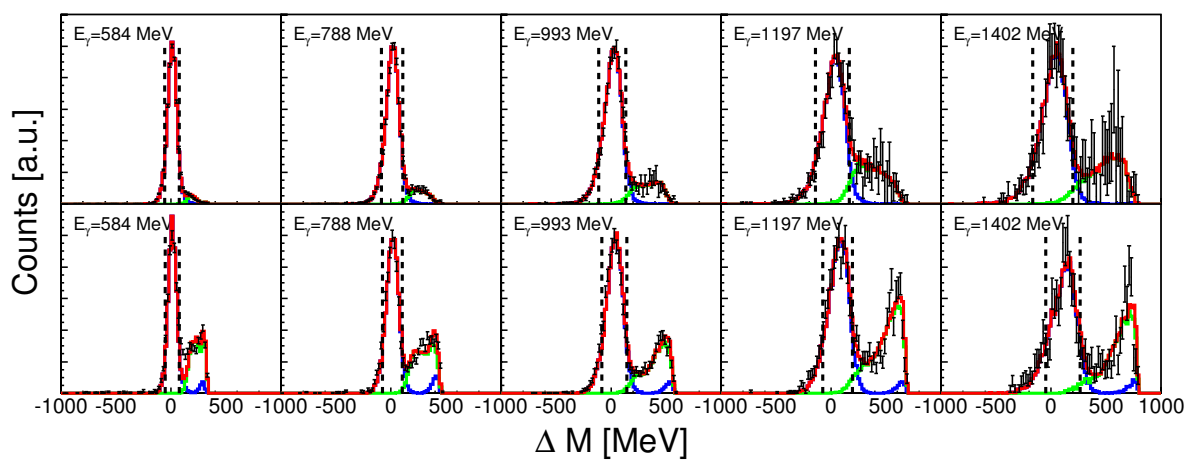

Figure 3. Missing mass distributions as function of the incident photon energy for exclusive $\pi^{0}$ photoproduction off the quasi-free neutron. Upper row: $-0.4<\cos \left(\theta_{\pi^{0}}^{*}\right)<-0.2$, lower row: $0.6<\cos \left(\theta_{\pi^{0}}^{*}\right)<0.8$. Black dots with error bars: Data, blue line: MC signal, green line: sum of MC background contributions, red line: sum of MC signal and MC background, dashed vertical lines: $1.5 \sigma$ cut positions.

production. The first measurement of the above mentioned reaction in quasi-free kinematics using a deuteron target was reported from the Crystal Ball/TAPS experiment at MAMI [15]. After the invariant mass cut for the $\pi^{0}$ meson, the reaction $\gamma N \rightarrow N \pi^{0}$ was identified with a missing mass technique. The spectra for the quasi-free proton are as expected very clean (since the proton is easily identified), but for the neutron it is more difficult to identify the reaction cleanly (because in the Crystal Ball no direct separation of neutrons and photons is possible so that the analysis must be based on combinatorial methods using the invariant mass of the decaying mesons. Typical results for the reaction identification off quasi-free neutrons are shown in figure 3. The results (total cross section, differential cross section and the comparison to existing theoretical models) of a high statistics measurement of the $\pi^{0}$ photoproduction off quasi-free protons and neutrons bound in the deuteron with the Crystal Ball/TAPS setup at the MAMI electron accelerator facility are published in [15] and is shown in figure 4.

\section{5 -}

The isospin decomposition for photoproduction of the isoscalar $\eta$-meson involves only two amplitudes: the isoscalar and the isovector ones, see equation 2.

$$
A(\gamma p \rightarrow \eta p)=A^{I S}+A^{I V} \quad A(\gamma n \rightarrow \eta n)=A^{I S}-A^{I V}
$$

Therefore the measurement of both reaction channels is equally important. Due to the isoscalar nature of the $\eta$ only $N^{\star}$ resonances couple to this decay channels and due to the large mass of the $\eta$ meson only a few partial waves contribute at medium incident energies. In the case of a free proton target the total cross sections and angular distributions have been studied with very high statistic quality. These results have been published in ELSA [17-19] and MAMI [20].

The study of the photoproduction off quasi-free neutrons gained a lot of interest, after the finding of an unexpected narrow structure in the excitation function [16]. The first dedicated experiment with high statistic has been performed by our group at ELSA using the Crystal Barrel/TAPS detector. The results $[21,22]$ confirm the peak at $\mathrm{W}_{R} \approx(1665 \pm 5) \mathrm{MeV}$, a width of $\Gamma_{R} \approx(25 \pm 15) \mathrm{MeV}$. A second generation measurement done at the MAMI accelerator with the Crystal Ball/TAPS detector 

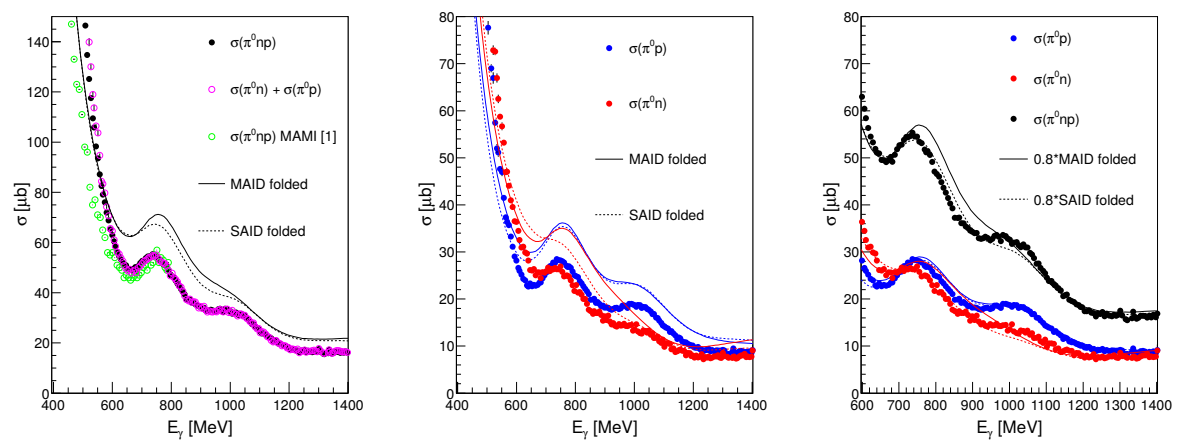

Figure 4. The total cross sections. Left: Full black dots: $\pi^{0} p n$, open magenta dots: $\pi^{0} p n=\pi^{0} p+\pi^{0} n$, open green dots: MAMI $1999 \pi^{0} p n$, black (dashed) line: MAID model (SAID analysis) Result folded with Fermi motion. Center: Full blue dots: $\pi^{0} p$, full red dots: $\pi^{0} n$, full (dashed) lines: MAID Model (SAID analysis) results folded with Fermi motion. Right: Full black dots: $\pi^{0} p n$, full blue dots: $\pi^{0} p$, full red dots: $\pi^{0} n$, full (dashed) lines: MAID model (SAID analysis) results folded with Fermi motion and scaled down with a factor 0.8 .

provided much higher statistical precision so that detailed studies of the angular distributions became available. The results showed a non-trivial angular dependence of the cross section, which cannot easily be reconcealed with the assumption that a narrow $P_{11}$ resonance interferes with the broad $S_{11}$ contributions [23]. Moreover, in order to demonstrate that nuclear effects like final state interactions play no significant role for this structure we also analysed data from a ${ }^{3} \mathrm{He}$ target [24]. In this case, the reaction kinematics is different due to the different momentum distributions of nucleons bound in the deuteron, respectively in ${ }^{3} \mathrm{He}$. The results show a resonance like structure at the same invariant mass (within the systematic uncertainty) and with the same width, see figure 5 .
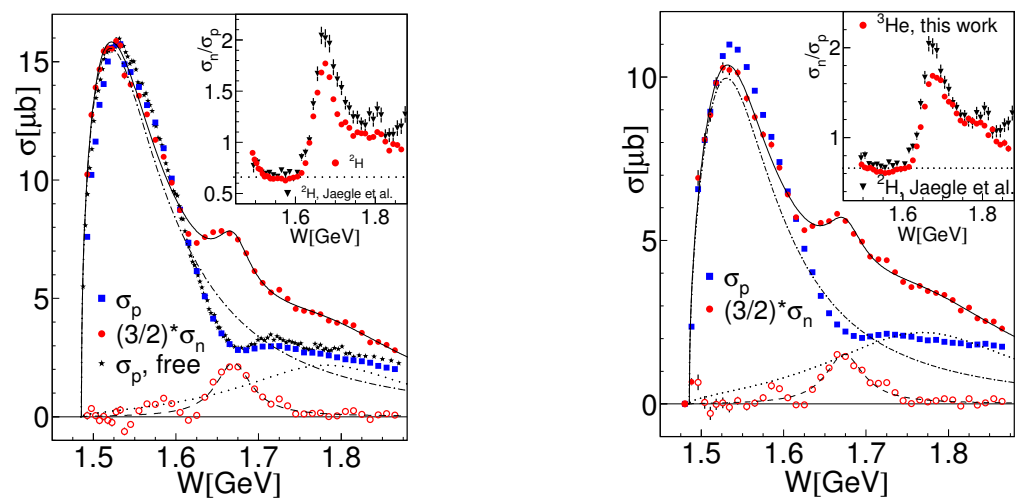

Figure 5. The quasi-free excitation functions for $\gamma N \rightarrow N \eta$. Left: deuteron [23] and free proton [20] targets; quasi-free proton (blue squares), free proton (black stars), and quasi-free neutron (red circles). Curves: fits (see text): $S_{11}$ contribution (dash-dotted), phenomenological background (dotted), narrow structure (dashed), sum of all (solid). Open red circles: data after subtraction of $S_{11}$ and background fit. Right: same for quasi-free neutron bound in ${ }^{3} \mathrm{He}$ target [24]. 
Starting from 2011, we have performed first measurements of the helicity dependence Eq. (3) of the photoproduction cross section of $\eta$ mesons off quasi-free protons and neutrons for photon energies from reaction threshold up to $1.6 \mathrm{GeV}$ at MAMI and $2.4 \mathrm{GeV}$ at ELSA, covering nearly the full solid angle. A large differences between measured data and theoretical predictions are observed which can be explained by a differences in the helicity amplitudes of well known and established resonances. The preliminary results were shown during the MESON'2014 conference with the very first MAID calculations. First results for polarization observables for this reactions will follow soon (data under analysis).

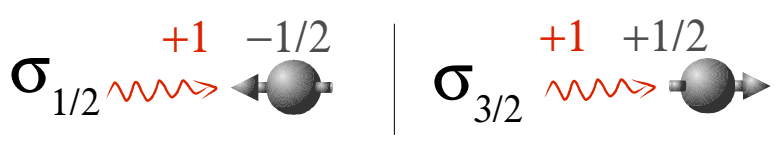

Figure 6. Circularly polarized photons and longitudinally polarized target

$$
\frac{d \sigma}{d \Omega}=\frac{d \sigma_{0}}{d \Omega}\left(1 \pm P_{T} P_{\odot} E\right) \quad E=\frac{N_{1 / 2}-N_{3 / 2}}{N_{1 / 2}+N_{3 / 2}} \frac{1}{P_{\odot} P_{T}} \frac{1}{d}
$$

\section{Photoproduction of meson pairs}

Photoproduction of meson pairs (like $\pi \pi$ and very first tries of $\pi \eta$ ) has been previously studied at MAMI and ELSA for different final states, measuring total cross sections, invariant mass distributions, and partly also the helicity dependence of the reaction cross section [25, 26]. However, the complicated amplitude structure of this reaction requires the investigation of more polarization observables. Such a program is currently active at MAMI and ELSA. The first results can be seen for the beam-helicity asymmetry for $\pi^{0} \pi^{0}$ and $\pi^{0} \pi^{+/-}$final states [27, 28]. More recently, due to technical progress in the experiments, the $\eta \pi$ final state became measurable. Total cross sections, an invariantmass distributions, and also some polarization observables have been measured for $\gamma p \rightarrow p \pi^{0} \eta$ [2932]. Data for all four isospin channels ( $\gamma p \rightarrow p \pi^{0} \eta, \gamma n \rightarrow n \pi^{0} \eta$ and $\gamma p \rightarrow n \pi^{+} \eta, \gamma n \rightarrow p \pi^{-} \eta$ ) has been measured at MAMI. The $\eta \pi$ final state is dominated by the decay cascade via $D_{33}(1700)$ resonance and simple predictions can be made for the cross section ratios of the different charge states. The electromagnetic helicity couplings for the excitation of $\Delta$ resonances are identical for protons and neutrons and from the Clebsch-Gordon coefficients can be estimated. The preliminary results for the total cross sections and their ratios were presented and compared to the simple calculation during the conference.

\section{Acknowledgements}

The results summarized in this work are part of the experimental programs of the A2 (MAMI) and CBELSA/TAPS (ELSA) collaborations. The presented results are parts of the $\mathrm{PhD}$ theses of M. Dieterle, D. Werthmüller, L. Witthauer, M. Oberle, A. Käser from the group of Prof. B. Krusche, Department of Physics, University of Basel. This work was supported by Schweizerischer Nationalfonds (200020132799,121781,117601,113511), Deutsche Forschungsgemeinschaft (SFB 443, SFB/TR 16), DFG-RFBR (Grant No. 05-02-04014), UK Sci- ence and Technology Facilities Council, (STFC 57071/1, 50727/1), European Community-Research Infrastructure Activity (FP6), the US DOE, US NSF and NSERC (Canada). 
MESON 2014-13 ${ }^{\text {th }}$ International Workshop on Production, Properties and Interaction of Mesons

\section{References}

[1] H. Herminghaus et al., IEEE Trans. Nucl. Sci., 30, 3274, (1983).

[2] K.-H. Kaiser et al., Nucl. Instrum. \& Methods A, 593, 159, (2008).

[3] W. Hillert et al., Eur. Phys. J. A28S1, 139 (2006).

[4] I. Anthony et al., Nucl. Instrum. \& Methods A, 301, 230, (1991).

[5] S. J. Hall et al., Nucl. Instrum. \& Methods A, 368, 698, (1996).

[6] J. C. McGeorge et al., Eur. Phys. J. A, 37, 129, (2008).

[7] D. Watts., Proc. of the 11th International Conference on Calorimetry in Particle Physics, Perugia, Italy, 2004, World Scientific, Singapore, 2005, (2005).

[8] A. Starostin et al., Phys. Rev. C, 64, 055205, (2001).

[9] R. Novotny., IEEE Trans. Nucl. Sci., 38, 379, (1991).

[10] A. R. Gabler et al., Nucl. Instrum. \& Methods A, 346, 168, (1994).

[11] E. Aker et al., Nucl. Instrum. \& Methods A, 321 , 69 (1992).

[12] G. Suft et al., A scintillating fibre detector for the Crystal Barrel experiment at ELSA. Nucl. Instrum. \& Methods A, 538 (416-424), September 2005.

[13] B. Krusche et al., Eur. Phys. J. A 6 (1999) 309-324.

[14] R.L. Walker et al., Phys. Rev. 182, 1729 (1969).

[15] M. Dieterle et al., Phys. Rev. Lett. 112, 142001.

[16] V. Kuznetsov et al., Phys. Lett. B 647, 23 (2007).

[17] V. Crede et al., Phys. Rev. Lett. 94, 012004 (2005).

[18] O. Bartholomy et al., Eur. Phys. J. A 33, 133 (2007).

[19] V. Crede et al., Phys. Rev. C 80, 055202 (2009).

[20] E.F. McNicoll et al., Phys. Rev. C 82, 035208 (2010).

[21] I. Jaegle et al., Phys. Rev. Lett. 100, 252002 (2008).

[22] I. Jaegle et al., Eur. Phys. J. A 47, 89 (2011).

[23] D. Werthmüller et al., Phys. Rev. Lett. A 49, 154 (2013).

[24] L. Witthauer et al., Eur. Phys. J. 111, 232001 (2013).

[25] B. Krusche et al., Eur. Phys. J. A 6, 309 (1999).

[26] F. Zehr et al., Eur. Phys. J. A 48, 98 (2012).

[27] M. Oberle et al., Phys. Lett. B 721, 237 (2013).

[28] M. Oberle et al., Eur. Phys. J. A 50, 54 (2014).

[29] E. Gutz et al., Eur. Phys. J. A 35, 291 (2008).

[30] E. Gutz et al., Phys. Lett. B 687, 11 (2010).

[31] V. Kashevarov et al., Eur. Phys. J. A 42, 141 (2009).

[32] V. Kashevarov et al., Phys. Lett. B 693, 551 (2010). 